\title{
Performance assessment of MIMO systems under partial information
}

\author{
H. Xia* \\ P. Majecki
}

Abstract-Minimum variance (MV) can characterize the most fundamental performance limitation of a system, owing to the existence of time-delays/infinite zeros. It has been widely used as a benchmark to assess the regulatory performance of control loops. For a SISO system, this benchmark can be estimated given the information of the system time delay. In order to compute the MIMO MV benchmark, the interactor matrix associated with the plant may be needed. However, the computation of the interactor matrix requires the knowledge of Markov parameter matrices of the plant, which is rather demanding for assessment purposes only. In this paper, we propose an upper bound of the MIMO MV benchmark which can be computed with the knowledge of the interactor matrix order. If the time delays between the inputs and outputs are known, a lower bound of the MIMO MV benchmark can also be determined.

\section{INTRODUCTION}

The control loop performance benchmarking techniques have built on ideas used successfully in business benchmarking. The aim is to diagnose control loop performance and provide tools to determine:

1) The best achievable performance which will be treated as the performance benchmark.

2) The controller performance index (CPI) which is the ratio of the performance benchmark to the actual performance.

Based on $C P I$, it can be seen whether there is any opportunity to improve the performance of the loop. The ways in which the loop performance may be improved will be in the realm of controller design. Controller benchmarking has been an active research area for the recent ten years [1], [4]. This interest started with the work of Harris [2]. In his paper, Harris proposed the use of closed-loop data to evaluate and diagnose controller performance using the output variance under the minimum variance (MV) controller as a benchmark.

The SISO MV benchmark is useful as the absolute lower bound on the achievable control performance and is attractive for its simplicity and minimum required information - only the output data collected from the plant and the estimate of the process deadtime are needed. For a MIMO system, the interactor matrix was introduced as a multivariable generalisation of the SISO time delay term. Assuming the full knowledge of the plant, a MIMO MV benchmarking method is presented in [3].

This work is supported by EPSRC Grant GR/R65800/01

* Corresponding author: hao.xia@strath.ac.uk

The authors are with Industrial Control Center, Department of Electrical Engineering University of Strathclyde, Glasgow, G1 IXQ, The United Kingdom
A. Ordys
M. Grimble

With the information of the magnitude of the time-delay, it is relatively easy to estimate the MV benchmark for a SISO system. In the case of MIMO systems, in order to estimate the MV benchmark, we need to construct a new . signal by filtering the system output with the interactor matrix of the system. Although it is possible to estimate the interactor matrices from the closed loop data [3], [6], this makes the computation of the MV benchmark for MIMO systems more difficult. Assuming the order of the interactor matrix is known, a simple interactor of the same order is proposed as a substitute for the original interactor matrix and prove that the performance index thus computed is an upper bound. Although the result is suboptimal, the computation procedure can be greatly simplified. Furthermore, a lower bound of the MV benchmark can be computed when the delay information between inputs and outputs of the plant is available. These bounds can be used to assess the CPI of the current controller which indicates its current performance level .

The rest of the paper is organized as follows: The derivation of the MV benchmark for SISO and MIMO systems is briefly introduced in section 2 . Then the estimation of the upper/lower bound of the MV benchmark is presented in sections 3 and 4. Using the FCOR technique introduced in [3], we illustrate the results on a simulated example in section 5. The paper is concluded in section 6. Due to space limitation, most of the proofs are omitted from the paper. A more detailed report can be obtained from the author upon request.

\section{MV CONTROLLER AND BENCHMARKING}

In this paper, our major focus is on the system performance in the steady state, and without loss of generality it is assumed in the following that the reference signal is set to zero. The only input to the system $\zeta_{t}$ is a zero-mean white noise of unity variance. The plant is modeled as:

$$
y_{t}=q^{-k} T u_{t}+N \zeta_{t}
$$

where $k$ is the time delay, $T$ is the delay-free plant transfer function, and $N$ is the disturbance transfer function. In the following subsections, the SISO MV controller for the plant (1) is first derived, then the result is generalised to MIMO systems. These derivations are standard and can be found in many references.

\section{A. The SISO MV controller}

Using the Diophantine identity:

$$
N=F+q^{-k} R=f_{0}+\cdots+f_{k-1} q^{-k+1}+q^{-k} R
$$


where $f_{i}$ (for $i=0, \cdots, k-1$ ) are constant coefficients, and $\mathrm{R}$ is the remaining rational, proper transfer function, Equation (1) can be rewritten as:

$$
y_{t}=F \zeta_{t}+q^{-k}\left(T u_{t}+R \zeta_{t}\right)
$$

The first term in this equation cannot be affected by the control action, i.e. $\operatorname{Var}\left(y_{t}\right) \geq \operatorname{Var}\left(F \zeta_{t}\right)$. The minimum variance control is achieved when the second term of equation (2) is set to zero, i.e.

$$
T u_{t}+R \zeta_{t}=0
$$

This yields

$$
u_{t}=-\frac{R}{T} \zeta_{t}
$$

Substituting equation (4) into (3) yields

$$
\zeta_{t}=F^{-1} y_{t}
$$

Substituting (5) into (4) gives the minimum variance control law

$$
u_{t}=-\frac{R}{F T} y_{t}
$$

So the minimum variance feedback controller is

$$
C_{M V}=-\frac{R}{F T}
$$

This particular version of minimum variance control requires the plant to be minimum phase if the control law is to be stabilizing. A block diagram of the closed loop system is shown in Fig.1. It is obvious that $F$ is independent of

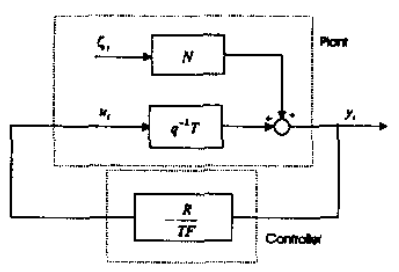

Fig. 1. Block diagram of a MV feedback control loop

the controller $C$, in other words the term $F \zeta_{t}$ which is the process output under minimum variance control, is feedback controller-invariant. The term $\operatorname{Var}\left(F \zeta_{t}\right)$ is defined as the MV benchmark.

If the transfer function from $\zeta_{t}$ to $y_{t}$ is modeled by an infinite moving-average (MA) time series model, then the computation of the MV benchmark $\operatorname{Var}\left(F \zeta_{t}\right)$ is equivalent to the estimation of the first $k$ terms of this model.

\section{B. The MIMO MV controller}

Time delay results in the most fundamental limitation on the achievable performance of any controller. Performance assessment of SISO processes as discussed before reflects this fundamental performance limitation in a stochastic framework. Wolovich and Falb [9] showed that the analog of the time-delay term in a SISO system is the interactor matrix in a MIMO system.
Theorem 2.1: For every $n \times m$ strictly proper, rational polynomial transfer-function matrix $T$, there is a unique, non-singular, $n \times n$ lower left triangular polynomial matrix $D$, such that $|D|=q^{r}$ and

$$
\lim _{q^{-1} \rightarrow 0} D T=\lim _{q^{-1} \rightarrow 0} \tilde{T}=K
$$

where $K$ is a full rank constant matrix. The integer $r$ is defined as the number of infinite zeros of $T$, and $\tilde{T}$ is the delay-free transfer-function matrix of $T$ which contains only finite zeros. The matrix $D$ is defined as the interactor matrix and can be written as

$$
D=D_{0} q^{d}+D_{1} q^{d-1}+\ldots+D_{d-1} q
$$

where $d$ denotes the order of the interactor matrix and is unique for a given transfer-function matrix, and $D_{i}$ (for $\mathrm{i}=0, \ldots, \mathrm{d}-1)$ are the coefficient matrices.

The interactor matrix $D$ can be assumed to be one of the three forms described in the sequel. If $D$ is of the form: $D=q^{d} I$, then the transfer function matrix $T$ is regarded as having a simple interactor matrix. If $D$ is a diagonal matrix, i.e., $D=\operatorname{diag}\left(q^{d_{1}}, q^{d_{2}}, \ldots, q^{d_{n}}\right)$, then $T$ is considered having a diagonal interactor matrix. Otherwise, $T$ is considered to have a general interactor matrix. A special type of general interactor matrix which is called the unitary interactor matrix was introduced in [7].

Definition 2.1: Instead of taking the lower triangular form, if an interactor matrix defined in Theorem 2.1 satisfies

$$
D^{T}\left(q^{-1}\right) D(q)=I
$$

then this interactor matrix is referred to as a unitary interactor matrix.

For any given full rank rational, proper transfer-function matrix $T$, there exists a non-unique unitary interactor matrix. However, it was shown' in [7] that any two unitary interactor matrices, $D(q)$ and $\tilde{D}(q)$, satisfy $\tilde{D}(q)=\Gamma D(q)$, $\Gamma^{T} \Gamma=I$. Here $\Gamma$ is an $n \times n$ unitary real matrix.

Using the interactor matrix, Huang and Shah [3] proposed a simple method of deriving the MIMO MV controller.

Consider a multivariable system

$$
Y_{t}=T U_{t}+N \zeta_{t}
$$

where $T$ is the system transfer function matrix and $N$ is the disturbance transfer function matrix. Then we have

Theorem 2.2: (Theorem 4.3.1 of [3]) For a multivariable process

$$
Y_{t}=T U_{t}+N \zeta_{t}
$$

where $\zeta_{t}$ is a vector of random white noise sources with zero mean, let $D$ be the interactor matrix of $T$ with the order $d$. The linear quadratic objective function defined by

$$
J_{M V}=E\left(\Phi_{t}^{T} \Phi_{t}\right)
$$

where $\Phi_{t}=q^{-d} D Y_{t}$ is minimized by an explicit optimal control law given by

$$
U_{t}=-\bar{T}^{-1} R F^{-1}\left(q^{-d} D\right) Y_{t}
$$


where $\grave{T}=D T, F$ and $R$ satisfy the Diophantine identity:

$$
q^{-d} D N=\underbrace{F_{0}+\ldots+F_{d-1} q^{-d+1}}_{F}+q^{-d} R
$$

and $R$ is a rational proper transfer function matrix. Furthermore, the MIMO MV benchmark is defined as

\section{Remarks:}

$$
J_{M V}=\operatorname{tr}\left[\operatorname{Var}\left(F \zeta_{t}\right)\right]
$$

- From above, it is clear that the interactor matrix is vital in the computation of MIMO MV benchmark if a data-driven method is preferred. According to (13) and (14), the benchmark can be the same for two different plants as long as they have the same interactor and disturbance transfer function matrix .

- If an MV controller is applied, the control signal is $u_{t}=-\frac{R}{T} \zeta_{t}$ for a SISO system and $U_{t}=-\bar{T}^{-1} R \zeta_{t}$ for a MIMO system. The control signal will be unbounded if $T$ or $\bar{T}$ contain non-minimum phase zeros.

- The inverse of $\bar{T}$ is used to derive the MIMO MV controller and this implies $n \leq m$, i.e. the number of plant inputs is greater or equal to the number of plant outputs. In another word, equation (12) in general only holds when $n \leq m$.

In the following, without loss of generality we assume that the plants can be represented as square strictly proper rational transfer-function matrices.

\section{THE UPPER BOUND OF THE MIMO MV BENCHMARK}

As discussed before, the interactor matrix is needed to estimate the MIMO MV benchmark. However, the computation of the interactor matrix is rather involved. Recursive algorithms for calculating a lower triangular and nilpotent interactor were proposed in [9] and [8], respectively. The need for a complete open loop transfer function limits the usefulness of these methods. It was later shown in [3] that the interactor matrix could be determined from the leading Markov parameter matrices of the plant model. Assuming the order of the interactor matrix is $d$, then the first $d$ Markov parameter matrices have to be found to calculate the exact interactor matrix. These matrices can only be obtained through open or closed-loop identification techniques.

In this section, it will be shown that an upper bound of the benchmark can be estimated based only on the information on the order of the interactor matrix.

Theorem 3.1: Let $J=\operatorname{tr}\left[\operatorname{Var}\left(F \zeta_{t}\right)\right]$ where $F$ satisfies the following identity:

$$
q^{-d} D N=\underbrace{F_{0}+\ldots+F_{d-1} q^{-d+1}}_{F}+q^{-d} R
$$

where $D$ is any unitary interactor matrix of order $d$ and $N=\sum_{i=0}^{\infty} N_{i} q^{-i}$ is a given transfer function. Then $J$ is maximised when $D=q^{d} I$.
Proof: Without loss of generality, it is assumed that $\zeta_{t}$ is a vector of zero mean white noise and $\operatorname{Var}\left(\zeta_{t}\right)=I$, then we have

$$
J=\operatorname{tr}\left[\operatorname{Var}\left(F \zeta_{t}\right)\right]=\operatorname{tr}\left[\sum_{i=0}^{d-1} F_{i}^{T} F_{i}\right]
$$

Since $D$ is a unitary interactor matrix of order $d$, it can be written as

$$
D=\sum_{i=0}^{d-1} D_{i} q^{d-i}
$$

Substituting $D$ and $N$ into the left hand side of (15), we have

$$
F_{i}=\sum_{j=0}^{i} D_{i} N_{i-j}, \quad i=0, \ldots, d-1
$$

Inserting (18) into (16), we have $J=\sum_{i=0}^{d-1} \sum_{j=0}^{d-1} \operatorname{tr}\left[G_{i j}\right]$ where $G_{i j}$ is the element of the following matrix

$$
G=\left(\begin{array}{ccc}
N_{0}^{T} \sum_{i=0}^{d-1}\left(D_{i}^{T} D_{i}\right) N_{0} & \cdots & N_{d-1}^{T} D^{T}{ }_{0} D_{d-1} N_{0} \\
N_{0}^{T} \sum_{i=0}^{d-2}\left(D^{T}{ }_{d-i-1} D_{i}\right) N_{1} & \cdots & \vdots \\
\vdots & \ddots & \vdots \\
N_{0}^{T} D^{T}{ }_{d-1} D_{0} N_{d-1} & \cdots & N_{d-1}^{T} D^{T}{ }_{0} D_{0} N_{d-1}
\end{array}\right)
$$

Based on the fact that $D$ is a unitary interactor matrix, i.e. $D^{T}\left(q^{-1}\right) D(q)=I$, we have

$$
\begin{aligned}
& \sum_{i=0}^{j} D_{d-i-1}^{T} D_{i}=0 \text { with } j=0, \ldots, d-2 \\
& \sum_{i=0}^{d-1} D^{T}{ }_{i} D_{i}=I
\end{aligned}
$$

Substituting (20) into $G, G$ can be rewritten as:

$G=\left(\begin{array}{cccc}N_{0}^{T} N_{0} & 0 & \cdots & 0 \\ 0 & N_{1}^{T} \sum_{i=0}^{d-2}\left(D_{i}^{T} D_{i}\right) N_{1} & \cdots & \vdots \\ \vdots & \vdots & \vdots & \vdots \\ 0 & N_{1}^{T} D^{T}{ }_{d-2} D_{0} N_{d-1} & \cdots & N_{d-1}^{T} D^{T} D_{0} N_{d-1}\end{array}\right)$

By careful inspection, it can found that the term $D_{d-1}$ does not appear in (21). Using the Lagrange multiplier technique [5], it can be shown that $J$ is maximised when $D_{d-1}$ is set to zero. Inserting $D_{d-1}=0$ back to (20), we have

$$
\begin{aligned}
& \sum_{i=0}^{j} D^{T-i-2} D_{i}=0 \text { with } j=0, \ldots, d-3 \\
& \sum_{i=0}^{d-2} D^{T}{ }_{i} D_{i}=I
\end{aligned}
$$

Substituting (22) back to (21), we have

$$
G=\left(\begin{array}{cccc}
N_{0}^{T} N_{0} & 0 & \cdots & 0 \\
0 & N_{1}^{T} N_{1} & \cdots & 0 \\
\vdots & \vdots & \ddots & \vdots \\
0 & 0 & \cdots & N_{d-1}^{T} D^{T}{ }_{0} D_{0} N_{d-1}
\end{array}\right)
$$


while $D_{d-2}$ does not appear in (23). As a result $J$ is maximised when $D_{d-2}$ is zero. Following the same line of thought, it can be found that $J$ is maximised with

$$
D_{0}^{T} D_{0}=I \quad D_{i}=0, i=1, \ldots, d-1
$$

If we set $D_{0}=I$, we can see that $J$ is maximised when $D=q^{d} I$.

Based on the above theorem, the following corollary can be obtained:

Conllary 3.1: Given the order of the interactor matrix is $d$, the least conservative upper bound of $J_{M V}$ can be found by estimating $J_{\text {upper }}=\operatorname{tr}\left(\operatorname{Var}\left(F \zeta_{t}\right)\right)$ where $F$ satisfies the following identity:

$$
N=\underbrace{N_{0}+\ldots+N_{d-1} q^{-d+1}}_{F}+q^{-d} R
$$

\section{THE LOWER BOUND OF THE MIMO MV BENCHMARK}

An upper bound of the MIMO MV benchmark has been discussed in the previous section. In this section, a lower bound will be introduced.

Given a transfer function matrix $T$, if the time delays between inputs and outputs are known, then a diagonal delay matrix associated with $T$ can be defined as:

$$
D_{d}(T)=\operatorname{diag}\left\{q^{d_{1}}, \ldots, q^{d_{n}}\right\}
$$

where each element $d_{i}$ of $D_{d}(T)$ is the minimum delay in the $i^{\text {th }}$ row of $T$. Another parametric matrix $U(T)$ can be defined as

$U(T)_{i, j}= \begin{cases}u_{i, j} \neq 0 & \text { if time delay of } T_{i, j} \text { equals } d_{i} \\ 0 & \text { otherwise }\end{cases}$

and $u_{i, j}$ represents the $i j^{\text {th }}$ element of $\lim _{q^{-1} \rightarrow 0} D_{d} T$

According to the definition of the interactor matrix (see (8)), we have the following lemma:

Lemma 4.1: For a transfer function matrix $T$ with only the input/output time-delay information given, if $\operatorname{det}(U(T)) \neq 0$ for all $u_{i, j} \neq 0$ then $D_{d}(T)$ is the diagonal interactor matrix of $T$.

Note that the above lemma is only a sufficient condition for $D_{d}(T)$ to be the diagonal interactor matrix of $T$. If there exists a set of $u_{i, j}$ s such that $\operatorname{det}(U(T))=0$, then the method introduced in [3] has to be used to find the interactor matrix of $T$.

Lemma 4.2: Given a transfer function matrix $T$, a diagonal polynomial matrix is defined as:

$$
\tilde{D}=q^{-1} D_{d}(T)
$$

where $D_{d}(T)$ is the diagonal delay matrix of $T$. Then the unitary interactor matrix $D$ of $T$ can be represented as:

$$
D=P \tilde{D}
$$

where $P$ is a unitary interactor matrix of $\tilde{D} T$.
Remarks: A useful fact worth being pointed out is that $\tilde{D}$ is only determined by the delay information of $T$, while $D$ can only be found with the full information of $T$.

Lemma 4.3: Let $J=\operatorname{tr}\left[\sum_{i=0}^{d-1} F_{i}^{T} F_{i}\right]$ where $F$ satisfies the following identity:

$$
q^{-d} D N=\underbrace{F_{0}+\ldots+F_{d-1} q^{-d+1}}_{F}+q^{-d} R
$$

where $D$ is a unitary interactor matrix of order $d$.

For any given integer $d^{\prime} \geq d$, we have $J=$ $\operatorname{tr}\left[\sum_{i=0}^{d-1} F_{i}^{T} F_{i}\right]=\operatorname{tr}\left[\sum_{i=0}^{d^{\prime}-1} F_{i}^{\prime T} F_{i}^{\prime}\right]$ where $F^{\prime}$ satisfies the following identity:

$$
q^{-d^{\prime}} D N=\underbrace{F_{0}^{\prime}+\ldots+F_{d^{\prime}-1}^{\prime} q^{-d^{\prime}+1}}_{F^{\prime}}+q^{-d^{\prime}} R^{\prime}
$$

Lemma 4.4: Given a transfer function matrix $T$, let $D_{d}(T)$ be the diagonal delay matrix of $T$. A set of unitary interactor matrices is defined as

$$
\mathcal{D}=\{D: D=P \tilde{D}\}
$$

where $P$ is any unitary interactor matrix and $\tilde{D}=$ $q^{-1} D_{d}(T)$.

For any $D \in \mathcal{D}$, a cost function is defined as $J(D)=$ $\operatorname{tr}\left[\sum_{i=0}^{d-1} F_{i}^{T} F_{i}\right]$ with $F$ satisfies the following identity:

$$
q^{-d} D N=\underbrace{F_{0}+\ldots+F_{d-1} q^{-d+1}}_{F}+q^{-d} R
$$

The order of $D$ is $d$ and $N=\sum_{i=0}^{\infty} N_{i} q^{-i}$ is a given transfer-function matrix. Then

$$
\arg \min _{D \in \mathcal{D}} J(D)=D_{d}(T)
$$

Based on the above lemmas, the following theorem is obtained concerning the lower bound of MIMO MV benchmark

Theorem 4.1: Given a transfer function matrix $T$, let $D_{d}(T)$ be its diagonal delay matrix with order $d$; the least conservative lower bound of $J_{M V}$ can be found by estimating $J_{\text {lower }}=\operatorname{Var}\left(F \zeta_{t}\right)$ where $F$ satisfies the following identity:

$$
q^{-d} D_{d}(T) N=\underbrace{F_{0}+\ldots+F_{d-1} q^{-d+1}}_{F}+q^{-d} R
$$

The above theorem can be interpreted as follows: the diagonal delay matrix can be considered as a simple generalization of the time delay of the SISO system. Let $N_{i}$ be the stochastic noise acting on the ith system output:

$$
N_{i}=\underbrace{f_{0} \xi_{t}+f_{1} \xi_{t-1}+\ldots+f_{d_{i}-1} \xi_{t-d_{i}-1}}_{e}+\ldots
$$

where $\xi_{t}$ is white noise and $d_{i}$ is the minimum time delay of the $i^{\text {th }}$ row of $T$.

It is obvious that $e$ is the portion of noise which is independent of feedback control. Furthermore there may be other portion of $N_{i}$ which cannot be compensated due to the other infinite zeros of $T$. This inevitably increases the achievable minimum variance. 


\section{EXAMPLES}

In the following, the application of the results obtained in the previous sections is demonstrated for performance assessment on a two by two MIMO controller. The approach consists of estimating the upper and lower bounds of the controller performance rather than the index itself. The advantage, however, is that it is not necessary to know the interactor matrix - the knowledge of the order of the interactor matrix and of the individual time delays is sufficient.

Example This example was originally used by Huang and Shah [3] to demonstrate the application of the FCOR algorithm to performance assessment of multivariable systems with the general interactor matrix. Our objective here will be to illustrate how Theorems (3.1) and (4.1) can be used to estimate the upper and lower bounds of the controller performance index from plant data, the interactor order $d$ and the individual time delays. For that purpose, we will apply the benchmarking algorithm twice: first assuming a simple interactor of order $d$, and then using the knowledge of the individual time delays to replace the actual interactor with its diagonal approximation. For comparison, we will also use full knowledge of the plant model to calculate the true general interactor matrix and hence the actual controller performance index.

The process has two inputs and two outputs and is described by the equation (10), with the plant and disturbance transfer matrices given as:

$$
\begin{aligned}
& T=\left[\begin{array}{cc}
\frac{q^{-1}}{1-0.4 q^{-1}} & \frac{K_{12} q^{-2}}{1-0.1 q^{-1}} \\
\frac{0.3 q^{-1}}{1-0.1 q^{-1}} & \frac{q^{-2}}{1-0.8 q^{-1}}
\end{array}\right] \\
& N=\left[\begin{array}{cc}
\frac{1}{1-0.5 q^{-1}} & \frac{-0.6}{1-0.5 q^{-1}} \\
\frac{0.5}{1-0.5 q^{-1}} & \frac{1.0}{1-0.5 q^{-1}}
\end{array}\right]
\end{aligned}
$$

The white noise input $a_{t}$ is a two-dimensional white noise sequence of the covariance matrix $\Sigma_{\zeta}=I$. Setting $K_{12}=1$ in the plant transfer matrix, the actual unitary interactor matrix for this example is of order $d=2$ and can be determined as

$$
D=\left[\begin{array}{cc}
-0.9578 q & -0.2873 q \\
-0.2873 q^{2} & 0.9578 q^{2}
\end{array}\right]
$$

In this case, the order of the interactor matrix equals the largest time delay of the system, however it must be stressed that generally this does not have to be the case. The MV criterion to minimize is the sum of the variances of the two outputs: $J_{M V}=E\left[Y_{t}^{T} Y_{t}\right]$. The minimum achievable value of $J_{M V}$ is determined by the polynomial matrix $F$ in equation (13) and this can be obtained as:

$$
F=\left[\begin{array}{cc}
-1.1014 q^{-1} & 0.2874 q^{-1} \\
-0.1916-0.0958 q^{-1} & -1.1302-0.5651 q^{-1}
\end{array}\right]
$$

The theoretically achievable minimum variance then follows from equation (14) as $J_{M V}=2.9990$.

On the other hand, if instead of using the general interactor matrix $D$ we use its simple and diagonal approximations $D_{\text {upper }}=q^{2} I$ and

$$
D_{\text {lower }}=\left[\begin{array}{ll}
q & 0 \\
0 & q
\end{array}\right]
$$

the corresponding polynomial matrices $F_{\text {upper }}$ and $F_{\text {lower }}$ will assume the form

$$
\begin{gathered}
F_{\text {upper }}=\left[\begin{array}{cc}
1+0.5 q^{-1} & -0.6-0.3 q^{-1} \\
0.5+0.25 q^{-1} & 1+0.5 q^{-1}
\end{array}\right] \\
F_{\text {lower }}=\left[\begin{array}{cc}
1 & -0.6 \\
0.5 & 1
\end{array}\right]
\end{gathered}
$$

and the upper and lower bounds of the minimum variance can then be calculated as $J_{M V}^{\text {upper }}=3.2896$ and $J_{M V}^{\text {lower }}=$ 2.6637 .

In reality, the plant and disturbance models might not be available and the above values have to be estimated from plant data. In order to estimate the minimum variance itself, the plant interactor matrix must be known. There are algorithms for its calculation or estimation from the first few Markov parameters of the plant [3], however they tend not to be very reliable in practice. Here we go around this problem by estimating the upper and lower bound of the minimum variance which only requires the knowledge of the order of the interactor matrix and of the individual time delays.

The FCOR (Filtering and Correlation) algorithm used in the numerical calculations is described in Huang and Shah [3]. The algorithm involves modeling the outputs as a multivariable time series in order to estimate the white noise driving sequences. This "whitening" step is not unique and may result in different polynomial matrices $F$ - in particular, the Cholesky algorithm can be used to obtain orthogonal driving sequences (i.e. of identity covariance matrix) that match the theoretical model. However, it is worth noting that the minimum achievable value of the cost function, i.e. the value that we eventually want to estimate, is invariant of the particular form of the polynomial matrix $F$. The estimates obtained (calculated using a data set of 5000 samples), together with the theoretical values, are given in Table I. An important point to note is that in order to

\begin{tabular}{|c|c|c|c|}
\hline & Lower Bound & Actual Value & Upper Bound \\
\hline Theoretical & 2.6637 & 2.9990 & 3.2896 \\
\hline Estimated & 2.5898 & - & 3.2426 \\
\hline
\end{tabular}

TABLE I

MINIMUM VARIANCE ESTIMATES 
estimate the coefficients of the polynomial matrix $F_{\text {tuper }}$, it was necessary to introduce for the time of the experiment an additional delay term to the controller transfer matrix. The reason for this can be explained briefly as follows. By assuming a simple interactor matrix of order $d$, we effectively attempt to estimate the first $d$ coefficient matrices of the disturbance model (2). Now with the actual plant (having a general interactor matrix) in the feedback loop, the closed-loop expression for the output is

$$
y_{t}=F \zeta_{t}+q^{-d} R \zeta_{t}-D^{-1} \bar{T} C_{0} y_{t}
$$

and in order to separate $F$ from the other terms on the right hand side of this equation (required for the FCOR algorithm), $d-1$ additional delays need be introduced to the controller $C_{0}$. On the other hand, there is no such difficulty when estimating the lower bound using the diagonal interactor.

To better see the effect which these approximations have on the calculated performance index, compare the three corresponding values for different values of parameter $K_{12}$ in the plant transfer matrix (this parameter determines the level of interaction between input 2 and output 1). The following multi-loop controller was used in all the simulations:

$$
K=\left[\begin{array}{cc}
\frac{0.5-0.2 q^{-1}}{1-0.5 q^{-1}} & 0 \\
0 & \frac{0.25-0.2 q^{-1}}{\left(1-0.5 q^{-1}\right)\left(1+0.5 q^{-1}\right)}
\end{array}\right]
$$

The $C P I$ is defined as $\frac{J_{M Y}}{E\left(\tilde{Y}_{t}^{T} \tilde{Y}_{t}\right)}$ where $\tilde{Y}_{t}$ is the system output $Y_{t}$ filtered by $q^{-d} D$. The results for different values of parameter $K_{12}$ are plotted in Figure 2. Considering
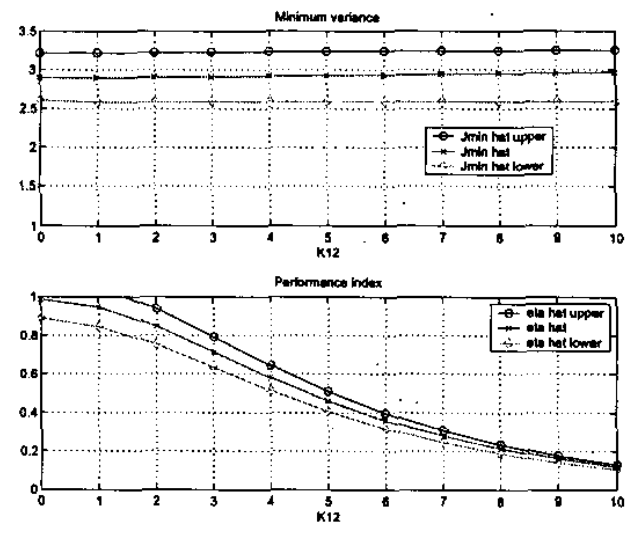

Fig. 2. Performance assessment of a MIMO system with the general interactor matrix

the definition of the CPI and Theorems (3.1) and (4.1), it is clear that the "true" performance index will always lie between the upper and lower bounds calculated based on these theorems. The price that must be paid for thus simplifying the problem is the necessity of introducing additional delays to the controller for the time of the benchmarking experiment (this concerns only the estimation of the upper bound). Moreover, the order of the interactor matrix needed for calculating the upper bound of the benchmark cost is not directly related to the actual delays present in the system and has to be determined separately.

\section{CONCLUSIONS AND FUTURE WORK}

In this paper, we have discussed controller performance assessment of multivariable systems using the minimum variance controller as a benchmark. In order to avoid the exact estimation of the interactor matrix, we proposed a method to estimate the upper and lower bound of the minimum achievable variance instead of the minimum variance itself. We proved that an upper bound of the MV benchmark can be estimated using only the known order of the interactor matrix, whereas the estimation of the lower bound requires only the knowledge of the individual time delays of the system. Although the knowledge of the interactor order is still a prerequisite, this considerably reduces the necessary information needed to assess the system performance.

The MV benchmarking procedures assess the performance of the existing controller against that of the optimal full-order controller. Such an unconstrained optimization problem results in high-order controllers (this order being at least as high as the order of the plant) and a question then arises how to adequately interpret the value of the calculated performance index: is it so low because the controller is poorly tuned or simply because it is not possible to get a better result with the existing controller structure? Future research will be focused on the computation of the meaningful benchmark under the controller structure constraints.

\section{REFERENCES}

[1] L. Desborough and T. Harris. Performance assessment measures for univariate feedback control. The Canadian Journal of Chemical Engineering, Vol.70:pp.1186-1197, 1992.

[2] T.J. Harris. Assessment of control loop performance. The Canadian Journal of Chemical Engineering, Vol.67:pp.856-861, 1989.

[3] B. Huang and S.L. Shah. Performance assessment of control loops: theory and applications. Springer Verlag, Berlin, 1999.

[4] B. Huang, S.L. Shah, and E.K. Kwok. Good, bad or optimal? performance assessment of multivariable processes. Automatica, Vol.33:pp.1175-1183, 1997.

[5] F.L. Lewis and V.L. Syrmos. Optimal control. John Wiley and Sons, New York, 1995.

[6] Y. Mutoh and R. Ortega. Interactor structure estimation for adaptive control of discrete-time multivariable nondecouplable systems. Automatica, Vol.29:pp.635-647, 1993.

[7] Y. Peng and M. Kinnaert. Explicit solution to the singular lq regulation problem. IEEE Transactions on Automatic Control, Vol.37:pp.633-636, 1992.

[8] M.W. Rogozinski, A.P. Paplinski, and M.J. Gibbard. An algorithm for the calculation of a nilpotent interactor matrix for linear multivariable systems. IEEE Transactions on Automatic Control, Vol.32:pp.234237, 1987.

[9] W. Wolovich and P. Falb. Invariants and canonical forms under dynamic compensation. SIAM Journal of Control and Optimization, Vol.14:pp.996-1008, 1976. 